\title{
La Resolución de Problemas Matemáticos y el Uso de Tecnología Digital en el Diseño de Libros Interactivos
}

\section{Mathematical problem solving and the use of digital technology for interactive book design}

\author{
Manuel Santos-Trigo*1 \\ msantos@cinvestav.mx \\ Matías Camacho-Machín** \\ mcamacho@ull.edu.es \\ *Cinvestav-IPN, México \\ **Universidad de La Laguna, España
}

\section{Resumen:}

Los libros de texto y los materiales de un curso de matemáticas siguen siendo un recurso importante para los estudiantes. En general, estos materiales ayudan a los estudiantes a revisar y extender sus conocimientos acerca de los temas que se estudian en la clase. Incluyen explicaciones de conceptos, definiciones y formas de resolver problemas, además de una lista de problemas variados que los estudiantes tienen que resolver. Los desarrollos de la tecnología digital están transformando el diseño y uso de los libros de texto en términos de ofrecer un material interactivo donde el estudiante pueda consultar vídeos que expliquen conceptos, revisar otros recursos en línea o compartir y discutir sus acercamientos con otros a través de aplicaciones usando una tableta o un teléfono móvil. En este artículo se presenta un marco, que incluye episodios que caracterizan el proceso de resolver problemas, para el diseño de materiales

\begin{abstract}
:
Textbooks are an essential resource that teachers use to organize and guide their students' learning of mathematics. In general, textbooks help students review and extend their knowledge of the themes that they study in their courses. Digital technology developments are transforming the ways in which textbooks are designed since this technology fosters the inclusion of interactive activities where students can consult videos to review and extend concepts, access and discuss online materials and share and discuss ideas or problem solving approaches within a learning community that include their peers or other students. In this article, we present a framework that aims to help teachers design interactive textbooks via the identification of main problem solving episodes such as understanding the problem statement and the construction of dynamic representations, looking for different ways to solve the problem, ex-
\end{abstract}

1 Dirección para correspondencia (correspondence address):

Manuel Santos-Trigo. Dpto. de Matemática Educativa, Cinvestav-IPN, Av. IPN 2508;

Sn. Pedro Zacatenco, 07360, CDMX (México). 
La Resolución de Problemas Matemáticos y el Uso de Tecnología Digital en el Diseño de Libros Interactivos

Manuel Santos-Trigo y Matías Camacho-Machín

interactivos donde los estudiantes tienen la oportunidad de representar y explorar problemas dinámicamente, formular conjeturas y relaciones y buscar argumentos que validen o sustenten esas relaciones.

\section{Palabras clave:}

Resolución de problemas; Sistemas de Geometría Dinámica (SGD); libros interactivos. tending the initial problem, and communicating results.

\section{Key words:}

Résolution des problèmes; Dynamic Geometry Systems (DGS); interactive books.

\section{Resumè:}

Les manuels sont des ressources essentielles que les enseignants utilisent pour organiser et guider l'apprentissage des mathématiques de leurs élèves. En général, les manuels aident les élèves à réviser et approfondir leurs connaissances sur les thèmes qu'ils étudient en cours. Les développements technologiques numériques transforment la façon dont les manuels sont conçus pour inclure des activités interactives où les étudiants peuvent consulter des vidéos pour réviser et étendre les concepts impliqués, accéder et discuter de matériel en ligne et partager et discuter d'idées ou d'approches de résolution de problèmes avec leurs pairs ou d'autres étudiants. Dans cet article, nous présentons un cadre qui vise à aider les enseignants à concevoir des manuels interactifs pour l'identification des principaux épisodes de résolution de problèmes: comprendre le problème et la construction de représentations dynamiques, chercher des solutions différentes pour résoudre le problème et communiquer les résultats.

\section{Mots clés:}

Problem solving; Systèmes de géométrie dynamique (SGD); livres interactifs

Fecha de recepción: 9-5-2018

Fecha de aceptación: 21-7-2018

\section{Introducción}

Un estudiante con el uso de tecnologías digitales consulta fuentes o recursos en línea para informarse y participa o contribuye en las discusiones de temas variados que se generan a través de las redes sociales. También es común que en sus tareas académicas revise materiales en línea, interactúe con sus pares y comparta ideas sobre cómo resolver un problema o comprender algún concepto matemático. Johnson, Coles \& Clarke (2017) afirman que las tareas matemáticas que trabajan los estudiantes moldean y caracterizan su aprendizaje. También sugieren que las oportunidades que tengan los estudiantes para desarrollar un pensamiento matemático dependen del tipo de tareas que se trabaje y lo que se valore y se promueva durante el proceso de resolución de las tareas. ¿Cómo el uso de tecnologías digitales incide en la formación de los 
estudiantes? ¿Qué transformaciones son importantes en los contenidos que se estudian en los ambientes escolares y en las formas de concebir y estructurar los escenarios de enseñanza? La irrupción de las tecnologías digitales en las actividades de los estudiantes demanda una discusión sistemática y sustentada sobre cómo incorporarlas en el estudio de las disciplinas y en los escenarios de enseñanza. ¿Seguirán siendo trascendentales los contenidos que se estudian en matemáticas y ciencias en la educación preuniversitaria? o ¿habrá una geometría distinta que incluya el estudio del movimiento de las figuras o un álgebra con poco énfasis en las operaciones o cálculos algebraicos y su significado? ¿Será todavía necesario que el niño en la educación primaria haga copias y copias para aprender a escribir? o ¿es mejor guiar al joven en el uso de los ordenadores y tabletas que lo ayuden a desarrollar o presentar un argumento estructurado y comprender lo que leen? ¿Desaparecerá el modelo de enseñanza donde, en general, un profesor, al frente de la clase, promueve actividades de aprendizaje en un ambiente controlado? ¿Deben considerarse desde ahora aquellos escenarios de enseñanza donde los estudiantes puedan ellos mismos diseñar su propio menú de aprendizaje que incluya una interacción en línea con otros estudiantes y asesores? La discusión de estas preguntas implica identificar qué tecnologías y desarrollos digitales son importantes en la construcción de conocimiento matemático de los estudiantes y caracterizar el tipo de razonamiento que pueden desarrollar durante sus experiencias de aprendizaje.

En este contexto, los materiales y libros de texto que involucran y demandan el uso sistemático de tecnologías digitales representan un medio para que el alumnado desarrolle formas de razonamiento consistentes con el quehacer de la disciplina. En este artículo se ilustra, como paso previo a la elaboración de un libro interactivo, la importancia de representar objetos y problemas dinámicamente, así como, las diversas exploraciones que resultan esenciales para que los estudiantes formulen conjeturas o relaciones matemáticas y busquen distintas maneras de sustentarlas.

\section{La importancia de los problemas y el uso de tecnologías digitales}

El diseño y las formas de implementar problemas o tareas matemáticas en los ambientes de aprendizaje son aspectos esenciales de la agenda académica en la educación matemática. Los problemas son el medio 
La Resolución de Problemas Matemáticos y el Uso de Tecnología Digital en el Diseño de Libros Interactivos

Manuel Santos-Trigo y Matías Camacho-Machín

para que los estudiantes se involucren en actividades que los lleven al desarrollo de un pensamiento matemático. En la construcción de un escenario de enseñanza resulta importante el diseño, la selección, o modificación de las tareas o problemas y también la observación y evaluación que involucra su implementación. Howson (2013) argumenta que los cambios en el currículum involucran repensar y diseñar nuevos materiales incluyendo libros de texto, guías para los profesores y analizar las formas de razonar que se promueven con el uso de diversas tecnologías digitales.

Los libros de texto no solo han sido una parte importante en el desarrollo del currículo, sino también han suministrado a los maestros un marco coherente para guiar su trabajo. ...buscan proveer a los maestros con una guía sobre el ritmo de su trabajo; $y$ han ayudado a definir las matemáticas que se enseñan y la cantidad de práctica y consolidación deseada a través de conjuntos de ejercicios sustentados (Howson, 2013, p. 648).

A partir del desarrollo notable de tecnologías digitales es necesario analizar qué tipo de problemas son importantes para que los estudiantes comprendan y construyan o desarrollen conocimiento matemático. Leung (2017) afirma que en "la educación matemática, cuando el salón de clase está inmerso en un ambiente de enseñanza y aprendizaje con tecnología, [es importarte preguntar] qué clase de matemáticas queremos que nuestros estudiantes aprendan, y también analizar cómo los profesores pueden usar la tecnología en el diseño de una enseñanza que valore la disciplina?" (p. 3-4). Es decir, resulta esencial que en el diseño de los problemas se aborde y señale el tipo de reflexión matemática que se intente promover en los estudiantes al usar la tecnología en la resolución de los problemas.

Existe un reconocimiento de que el uso de diversas tecnologías digitales puede ofrecer diversas oportunidades a los estudiantes en términos de analizar las formas de construir y explorar representaciones de los problemas, en los procesos de formulación de conjeturas y relaciones, en la búsqueda de argumentos para sustentar alguna conjetura y en las maneras de discutir y comunicar resultados.

Watson \& Ohtani (2015) afirman que el proceso de diseñar tareas o problemas es importante desde varias perspectivas: La cognitiva que considera la relación entre los contenidos de las tareas y las experien- 
cias previas de los estudiantes; la cultural que involucra la influencia de las tareas en la construcción de una conceptualización del quehacer matemático y su naturaleza; y la perspectiva práctica donde las tareas representan la esencia de lo que hace el estudiante en el salón de clase.

Leung (2017) también argumenta que "los profesores deben experimentar por ellos mismos, como estudiantes, el potencial y las dificultades [en el uso] de tecnología digital en el aprendizaje de las matemáticas, y así adquirir conocimiento acerca de cómo los estudiantes pueden aprender matemáticas en varios ambientes digitales" (p. 6). Pepin et al., (2017) afirman que en el ámbito internacional los profesores usan diversos recursos digitales en sus prácticas de enseñanza, pero la abundancia y disponibilidad de esos recursos les plantea retos en términos de evaluar su pertinencia y calidad, y posible integración sistemática y significativa en las clases.

En particular, el diseño e implementación de las tareas implica reflexionar acerca de los conceptos y contenidos involucrados en la tarea, los procesos asociados con la formulación de conjeturas, la búsqueda de diversas formas de resolverlas, las extensiones o generalizaciones, la construcción de modelos y las estrategias de resolución de los problemas.

En la implementación de las tareas, Schoenfeld (2008) argumenta sobre la importancia de poner atención y analizar los procesos involucrados en la resolución de las tareas y no solo a las respuestas o soluciones; y considerar diversas dinámicas de enseñanza que involucren la participación individual, de grupos pequeños y discusiones plenarias con toda la clase. Además, motivar a los estudiantes a que escuchen a sus pares, promover una actitud inquisitiva que los lleve a criticar y sustentar relaciones matemáticas e involucrar a los estudiantes en actividades donde tengan la oportunidad de expresar sus ideas y acercamientos en forma oral y escrita.

En este contexto, es fundamental identificar y analizar el tipo de tareas que se pueden diseñar a partir de un uso coordinado de diversas tecnologías y desarrollos digitales. ¿Qué tecnologías digitales ofrecen a los estudiantes oportunidades para construir conocimiento matemático y competencias de resolución de problemas? ¿Qué se promueve en el diseño de las tareas matemáticas a partir de los procesos matemáticos que los estudiantes pueden desarrollar en sus aproximaciones o soluciones? ¿Cómo implementar esas tareas en los escenarios de enseñanza? En la 
La Resolución de Problemas Matemáticos y el Uso de Tecnología Digital en el Diseño de Libros Interactivos

Manuel Santos-Trigo y Matías Camacho-Machín

discusión de estas preguntas se intenta caracterizar elementos importantes en el diseño de tareas matemáticas que involucren el uso coordinado de tecnologías digitales.

Los estudiantes en su formación académica deben construir conocimiento sólido y estrategias que los lleve a resolver problemas. En un ambiente de enseñanza, los libros de texto o materiales son esenciales para el desarrollo de las actividades que se llevan a cabo en las aulas. Pepin et al. (2017) identifica tres tipos de textos digitales: (i) la digitalización de un libro tradicional y que ahora presenta una serie de uniones o conexiones con otros objetos de aprendizaje que el maestro considera importantes para los estudiantes; (ii) un libro digital en constante revisión y desarrollo a partir de los comentarios recibidos por otros maestros; y (iii) el tipo de libro digital interactivo que se basa en un conjunto de objetos de aprendizaje que incluye tareas y herramientas que el estudiante usa en la comprensión de los conceptos y en la resolución de problemas. En este artículo el centro de atención será el diseño de un libro digital interactivo en el que se destacarán los modelos dinámicos de los problemas con el objetivo de que estos modelos faciliten el proceso de formular y sustentar conjeturas matemáticas.

\section{El movimiento de las figuras y objetos matemáticos y los libros digitales}

El desarrollo de los Sistemas de Geometría Dinámica (SGD) como GeoGebra representa un avance significativo, dado que proporciona a los estudiantes herramientas para representar y explorar conceptos y problemas matemáticos. Leung (2008) menciona que estos ambientes dinámicos resultan poderosos, ya que generan un micro-mundo para la adquisición del conocimiento matemático que permite visualmente hacer explícito el dinamismo implícito del pensamiento acerca de los conceptos matemáticos.

Una de las ventajas de un SGD es que nos provee de una habilidad para retener el contexto o esencia de una configuración geométrica mientras podemos traer al frente aquellas partes dinámicas de toda la configuración que nos interesan. Esto es, podemos visualizar el estudio de la variación de un aspecto de la configuración mientras mantenemos otros aspectos constantes, 
así anticipando la emergencia de patrones invariantes (Leung, 2008, p. 135).

Johnson et al. (2017) mencionan que las tareas o problemas son esenciales y representan el medio para que los estudiantes desarrollen y muestren un pensamiento matemático consistente con el quehacer de la disciplina. Así, un problema puede utilizarse para introducir un contenido matemático nuevo, consolidar un procedimiento enseñado, generar oportunidades para que los estudiantes apliquen un concepto o procedimiento en una situación nueva, evaluar la comprensión de un concepto, o crear condiciones para que los estudiantes desarrollen y muestren habilidades genéricas y de colaboración para resolver problemas. El uso de tecnologías digitales en el ámbito escolar hace posible diferentes formas de interacción entre los materiales, los estudiantes y el maestro. Con el uso sistemático de tecnologías digitales los problemas se conceptualizan como un punto de partida para involucrar a los estudiantes en una reflexión matemática continua.

Santos-Trigo \& Camacho-Machín (2013) proponen un marco sobre cómo transformar un ejercicio o problema rutinario en un conjunto de actividades que involucran una reflexión matemática. El marco incluye fases importantes relacionadas con el proceso de representar, explorar y resolver los problemas. La formulación de preguntas y la búsqueda de diversas maneras de responderlas facilita cada una de las fases que se destacan en el proceso de solución: (a) la comprensión de los enunciados del problema que involucra analizar los conceptos y la pertinencia y el sentido de la información y lo que se intenta resolver; (b) la representación o modelización del problema incluyendo aquí la construcción de un modelo dinámico de la situación; (c) el diseño y la exploración de un plan de solución; (d) la búsqueda de diferentes métodos de solución; (e) la extensión de los problemas y la formulación de nuevas preguntas; (f) y la comunicación de resultados.

Así, problemas situados en un contexto matemático o que involucren eventos o situaciones que se pueden modelizar y explorar vía el uso de recursos y objetos matemáticos, resultan importantes para que los estudiantes busquen diferentes maneras sobre cómo resolverlos. Es decir, no solo se busca que los estudiantes encuentren la solución de un problema; sino que siempre identifiquen y analicen varios caminos para resolverlos y se interroguen sobre los conceptos y estrategias que emergen en las diferentes formas de solución.

En la resolución de problemas con el uso sistemático de un SGD, surgen 
La Resolución de Problemas Matemáticos y el Uso de Tecnología Digital en el Diseño de Libros Interactivos

Manuel Santos-Trigo y Matías Camacho-Machín

de manera natural objetos matemáticos básicos como el triángulo, cuadrado, rectángulo, paralelogramos, y cuadriláteros que se estudian desde la Educación Primaria. ¿Qué referentes desarrollan y exhiben los estudiantes al resolver problemas que involucran propiedades y resultados asociados con esos objetos? El uso de tecnología digital en la representación y exploración de objetos matemáticos genera oportunidades a los estudiantes para investigar el comportamiento de los objetos y sus atributos en términos de significados geométricos y la búsqueda de relaciones. Es decir, el movimiento de los objetos permite a los estudiantes enfocar la atención hacia aquellos elementos o atributos, de esos objetos, que se mantienen invariantes y así plantear conjeturas sobre su comportamiento. La formulación de conjeturas también conduce a los estudiantes a la búsqueda de argumentos o propiedades para sustentar o validar esas conjeturas.

\section{Sobre el diseño y presentación de los Problemas: La importancia de la búsqueda de múltiples métodos de resolución}

Se presenta un ejemplo que necesita del estudio de propiedades de una figura que se estudia desde los niveles más elementales, el triángulo isósceles. En la construcción de este triángulo se muestra la importancia de explorar diferentes caminos para resolver un problema así como diferentes maneras de establecer conexiones entre distintos objetos matemáticos.

Problema: Si se tiene el perímetro de un triángulo isósceles y una de sus alturas, ¿Cómo se puede construir ese triángulo?

Comprensión del enunciado del problema: ¿Qué objetos matemáticos están involucrados en el problema? ¿Qué información o datos se dan? ¿Cómo se puede representar el perímetro y la altura de un triángulo? ¿Existe alguna relación entre estos dos elementos que ayude en la construcción del triángulo? ¿Cómo representar y explorar un modelo parcial dinámico del problema? ¿Existe información suficiente para construir el triángulo? La idea es que los estudiantes formulen este tipo de preguntas y después de una reflexión individual, las discutan en pareja o con grupos de tres. Esta discusión es importante ya que ayuda a los estudiantes a activar sus conocimientos sobre los conceptos o temas alrededor del problema. 
Modelos dinámicos del problema y la solución. Un aspecto relevante en el diseño de un libro interactivo es que los objetos o atributos matemáticos y problemas se representen en forma dinámica. La idea es que el modelo dinámico sea un referente para que el estudiante tenga oportunidad de buscar relaciones entre los objetos y sus atributos (medida de ángulos, perímetros, áreas, etc.). En este contexto, un problema representa un punto de partida y oportunidad para que los estudiantes se involucren en un razonamiento y reflexión matemática que los lleve a buscar diversos caminos para representar, explorar y resolver el problema. A continuación, se muestran varias aproximaciones que surgen en la tarea de representar y explorar el problema a través de un modelo dinámico.

A) La mediatriz. A partir de la interpretación geométrica de la información dada, una primera aproximación al problema se basa en construir un triángulo isósceles sobre un segmento que se puede asociar con el perímetro. Se enfoca la atención en la generación de una familia de triángulos isósceles que tienen la altura dada. Así, al mover uno de los vértices del triángulo sobre el segmento que representa el perímetro se busca la posición para que ese triángulo cumpla con las condiciones que se piden. En la figura 1 los segmentos AB y FG representan, respectivamente, el perímetro y la altura del triángulo isósceles que se desea construir. La recta EC es la mediatriz de AB, el segmento EC corresponde a la altura FG y D es un punto sobre el segmento $\mathrm{AE}$ y $\mathrm{D}^{\prime}$ es el simétrico de $\mathrm{D}$ con respecto al punto $\mathrm{E}$. El triángulo $\mathrm{DCD}^{\prime}$ es isósceles con altura $\mathrm{FC}=\mathrm{EC}$. La recta j es la mediatriz del segmento AC. Cuando el punto D coincide con la intersección de la mediatriz y el segmento $A B$ (punto $H$ ), se tiene que el triángulo $\mathrm{HCD}^{\prime}$ es isósceles y tiene como altura el segmento FG (Figura 2). Esto es por la definición de mediatriz se tiene que $\mathrm{AH}=\mathrm{HC}$.

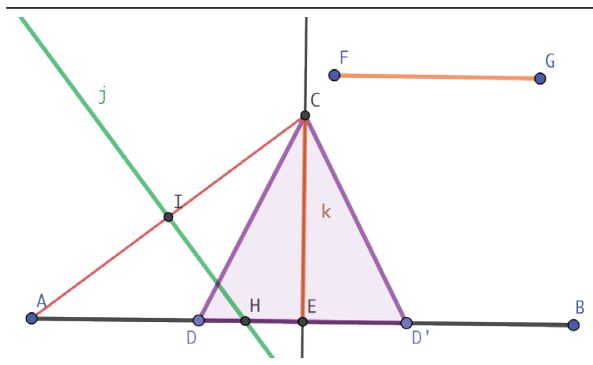

Figura 1. La mediatriz del segmento $A B$ en la búsqueda de la solución del problema. 
La Resolución de Problemas Matemáticos y el Uso de Tecnología Digital en el Diseño de Libros Interactivos

Manuel Santos-Trigo y Matías Camacho-Machín

Bastará, por tanto, con mover el punto D y cuando coincida con el punto $\mathrm{H}$ se obtendrá el triángulo isósceles que pide el problema. Se puede también consultar información sobre la mediatriz en:

http://mathworld.wolfram.com/PerpendicularBisector.html

B) Una Parábola. En esta aproximación se construye una familia de triángulos isósceles que tenga el perímetro dado y diferentes alturas. De esa familia se buscará aquél que tenga la altura deseada. En la figura 3, los segmentos $A B$ y $C D$ representan el perímetro y la altura dada. El punto E está sobre el segmento AM ( $M$ es punto medio de $A B)$. Se traza la mediatriz del segmento AE y una circunferencia de radio $\mathrm{EF}$ ( $\mathrm{F}$ es el punto medio del segmento $\mathrm{EB}$ ) y centro el punto $\mathrm{E}$. La circunferencia y la mediatriz se intersecan en el punto G. El triángulo AGE es isósceles y al mover el punto $E$ sobre el segmento $A B$ se genera una familia de triángulos isósceles. $A J$ representa la altura $C D$ y la recta $i$ es perpendicular al eje de ordenadas (OY) que pasa por J (Figura 3). El lugar geométrico del punto G que se genera al mover el punto $E$ sobre el segmento $A B$ es una parábola. La recta perpendicular $i$ y la parábola se intersecan en el punto $T$ y cuando $G$ coincide con $T$ el triángulo $A G E$ es isósceles y tiene como perímetro el segmento $A B$ y altura CD (Figura 4).

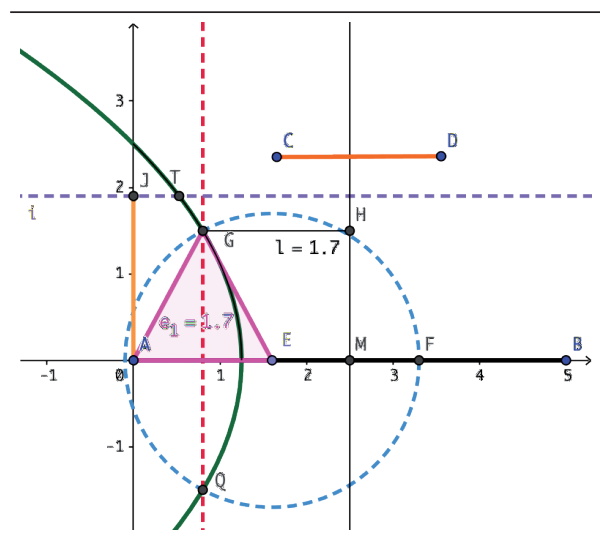

Figura 3. El lugar geométrico del punto $\mathrm{G}$ cuando el punto $\mathrm{E}$ se mueve sobre el segmento $\mathrm{AB}$ es una parábola

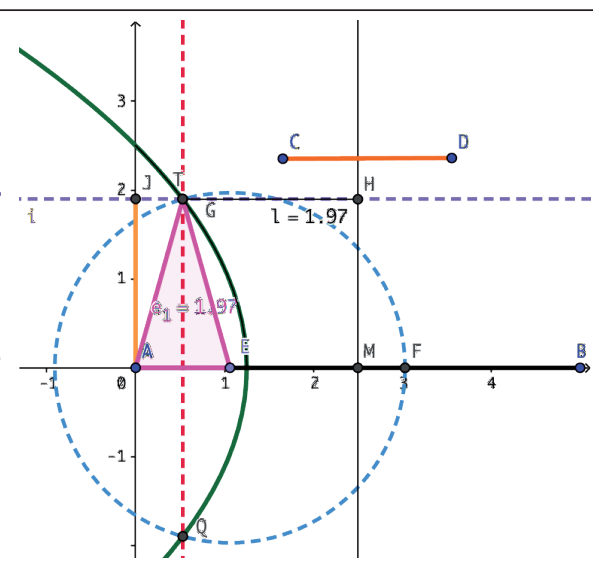

Figura 4. Al hacer coincidir el vértice $G$ con el punto $T$ se obtiene el triángulo isósceles con las condiciones del problema. 
Para mostrar que el lugar geométrico que genera $G$ cuando se mueve E representa una parábola, bastará observar que el foco es el punto $A$ y la directriz es la perpendicular al segmento $A B$ que pasa por el punto $M$ ( $M$ es el punto medio de $A B$ ) y como consecuencia $d(A, G)=d(G, H)$

C) Una elipse. El mismo problema se puede resolver con la generación de una elipse. En la figura 5, los segmentos $A B$ y $C D$ representan, respectivamente, el perímetro y la altura del triángulo isósceles que se desea construir. El punto $E$ es el punto medio del segmento $A B$ y $G$ es un punto sobre el segmento EB. Se traza la perpendicular al segmento $A B$ que pasa por el punto $E$ y dos circunferencias, una con centro en $E$ y radio $E G$ y otra con centro en $F$ (EF es la altura $C D$ ) y radio GB. Estas circunferencias se cortan en los puntos $\mathrm{H}$ e I y el lugar geométrico que describen estos puntos cuando el punto $\mathrm{G}$ se mueve sobre el segmento EB es una elipse (Figura 5). Los puntos de intersección de la elipse y el segmento $A B$ determinan el triángulo isósceles que cumple con las condiciones el problema.
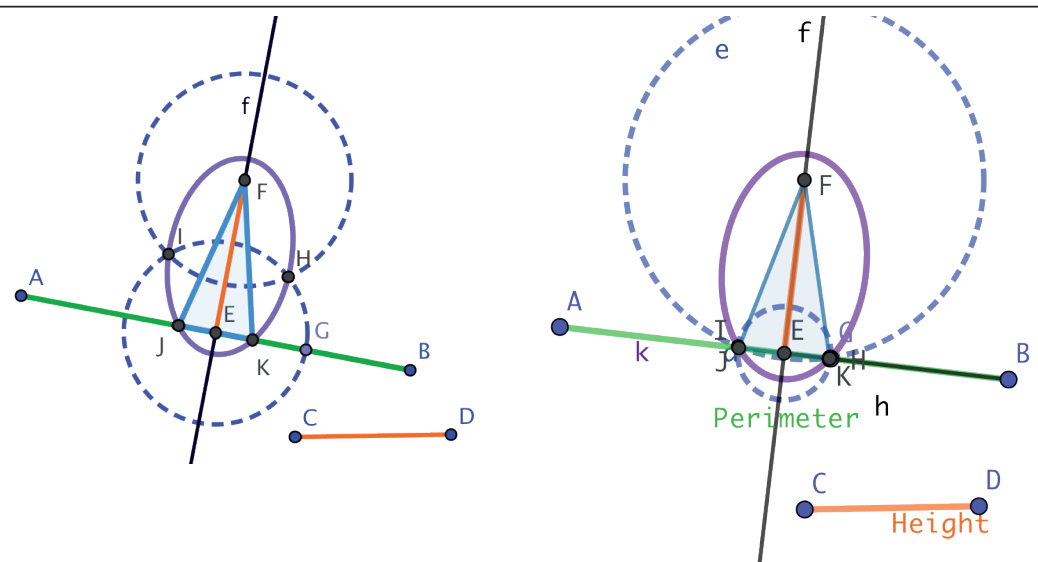

Figure 5. El lugar geométrico de los

puntos I \& H cuando G se mueve sobre EB es una elipse.

Figure 6. Triángulo JKF es isósceles con perímetro el segmento $\mathrm{AB}$ y tiene como altura el segmento CD.

Moviendo el punto G sobre el segmento EB, se puede observar que cuando los puntos $\mathrm{H}$ e I coincidan con $\mathrm{K}$ y J, respectivamente, se obtiene el triángulo isósceles que se pide. Se puede observar que los focos de la elipse son los puntos E y $\mathrm{F}$ y la constante es la suma de los radios de las dos circunferencias. 
D) Una hipérbola. En la Figura 7 los segmentos $A B$ y $C D$ representan el perímetro y la altura del triángulo isósceles que se desea construir. $\mathrm{E}$ es el punto medio del segmento $A B$ y $F$ un punto sobre el segmento $A E$. La recta $\mathrm{GH}$ es la mediatriz del segmento AF y el segmento $\mathrm{GH}$ tiene la misma longitud de la altura CD. El triángulo AFH es isósceles con altura $\mathrm{CD}$. El punto Q tiene como abcisa la coordenada $\mathrm{X}$ del punto $\mathrm{F}$ y como ordenada el perímetro del triángulo AFH. El lugar geométrico del punto $Q$ cuando el punto $F$ se mueve sobre $A E$ es una hipérbola que se puede validar a través de la herramienta que dibuja una cónica que pasa por cinco puntos del lugar geométrico. Consideremos el segmento AJ congruente con el segmento $A B$ (el perímetro). La intersección de la perpendicular al eje $Y$ que pasa por J y el lugar geométrico determina el triángulo isósceles pedido (Figura 8).

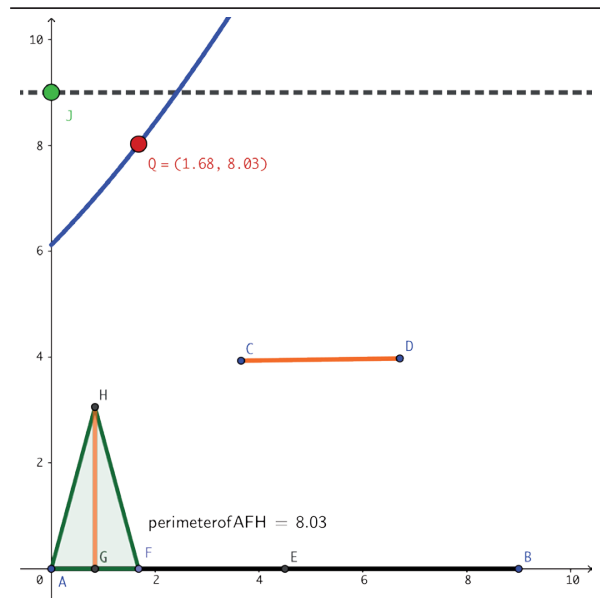

Figura 7. El lugar geométrico del punto $\mathrm{Q}$ representa la variación del perímetro cuando $\mathrm{F}$ se mueve sobre AE.

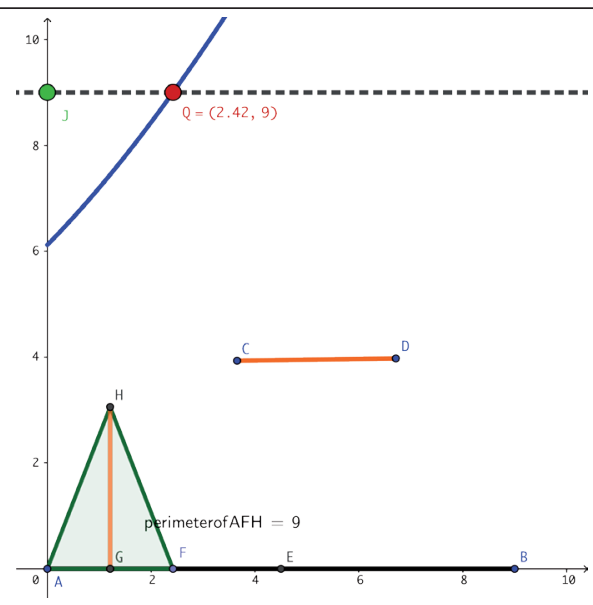

Figura 8. La intersección del lugar geométrico del punto Q y la perpendicular al eje OY determina la posición de F y del triángulo AFH con las condiciones del problema.

Se observa en la figura 8 que al mover el punto $\mathrm{F}$ sobre el segmento $\mathrm{AE}$, el punto $\mathrm{Q}$ se mueve y la solución se obtiene cuando $\mathrm{Q}$ pertenece a la recta perpendicular al eje de ordenadas (OY) que pasa por J.

Una aproximación algebraica. En la figura 9a se tiene el segmento que representa al perímetro $P$ y a la altura $h$ (datos del problema). Teniendo en cuenta esos datos, se obtendrá el siguiente sistema de 
ecuaciones por el Teorema de Pitágoras (1) y el significado del perímetro (2):

$$
\left\{\begin{array}{l}
l^{2}=h^{2}+r^{2} \ldots \ldots(1) \\
P=2 l+2 r \ldots \ldots(2)
\end{array}\right. \text {. }
$$

Al resolver el sistema de ecuaciones (1) y (2), se obtienen los valores de $l$ y $r$ en función del perímetro y la altura:

$$
r=\frac{P}{4}-\frac{h^{2}}{P} ; l=\frac{h^{2}}{P}-\frac{P}{4} .
$$

Para construir el triángulo se trazan dos circunferencias: Una centrada en un punto $A$ y de radio $2 r$ y la otra también con centro en $A$ y radio l. Se coloca un punto $B$ sobre la circunferencia de radio $2 r$ y se traza la mediatriz de $A B$. El punto $C$ es donde se interseca la mediatriz con la circunferencia de radio $\mathrm{l}$. El triángulo $\mathrm{ABC}$ cumple las condiciones del problema (Figura 9b).

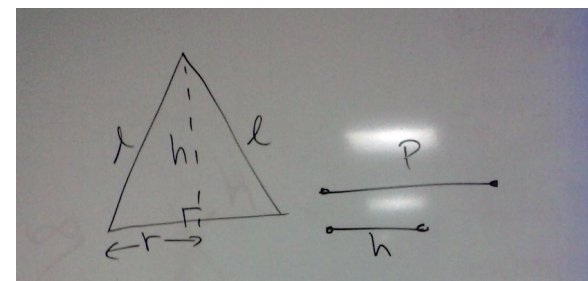

Figura 9a. Representación para resolver el problema con recursos algebraicos

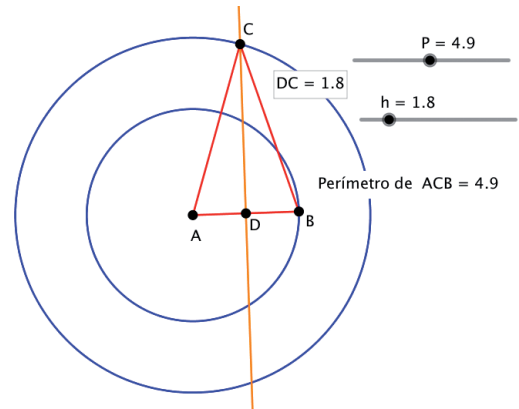

Figura 9b. El triángulo ACB cumple las condiciones del problema

Los diferentes caminos que surgen durante la construcción y exploración de un modelo dinámico del problema, nos llevan a interpretar esta primera fase como una de las más importantes de cara a resolver el problema y de diseñar el libro interactivo. Las situaciones que se observan constituyen un punto de partida para que en los restantes episodios (Santos-Trigo \& Camacho-Machín, 2013) que se desarrollarían posteriormente, el estudiante quede inmerso en un ambiente de actividad matemática esencial para el aprendizaje de conceptos y procedimientos matemáticos. 
La Resolución de Problemas Matemáticos y el Uso de Tecnología Digital en el Diseño de Libros Interactivos

Manuel Santos-Trigo y Matías Camacho-Machín

Una vez desarrollado este análisis y resolución del problema desde las diferentes aproximaciones, una forma de presentar el problema a los estudiantes será mediante un libro interactivo elaborado ad hoc y accesible en la plataforma de GeoGebra. En nuestro caso, en el siguiente enlace se podrá acceder al mismo: https://ggbm.at/KwUcxerf

La portada del libro se observa en la figura 10. Además de la introducción, se incorporan una serie de capítulos que, como se puede observar, recogen los diferentes episodios analizados en los párrafos anteriores que han permitido determinar diferentes perspectivas que enriquecen los conocimientos matemáticos que se derivan del trabajo interactivo que se facilita con él.

\section{Resolución de Problemas en un Libro Interactivo}

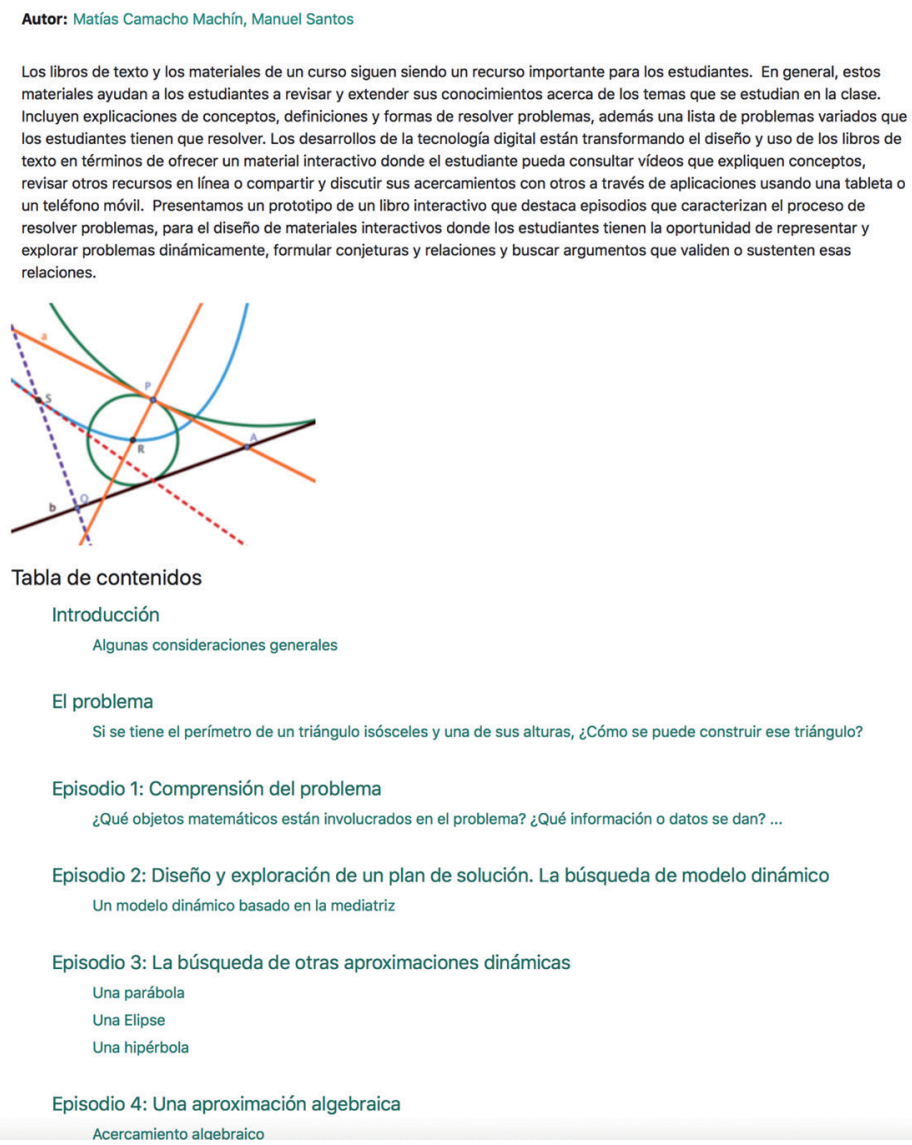

Figura 10. La portada del libro interactivo. 
El diseño de libros interactivos, basados en la resolución de problemas, atendiendo al marco que hemos definido, resulta ser un campo de investigación interesante y GeoGebratube no es la única plataforma que facilita su construcción, aunque esta es quizás la de más fácil acceso y más intuitivamente manejable. Estos recursos son interesantes ya que están acordes con los tiempos que corren, en los que la fácil disposición de diversas herramientas digitales favorecen su inclusión como un recurso importante. Pasamos a continuación a mostrar un ejemplo de libro interactivo ${ }^{2}$, diseñado por el primer autor, el cual incorpora diversas herramientas que se encuentran a disposición del usuario y que permiten incorporar applets, enlaces, etc.

Se presenta a continuación otro modelo de libro interactivo, pero elaborado para Ipad, lo que podría constituir un hándicap, dado que no todos los usuarios pueden disponer de ellos. Se incluye aquí uno de los dos problemas que se recogen en el libro interactivo creado en iBooks Author (iBA) para iPad, que lleva por título El Estudio de fenómenos de variación y el empleo de herramientas digitales ${ }^{3}$. En él se incluyen más de una decena de widgets que permiten al estudiante manipular y descubrir propiedades geométricas.

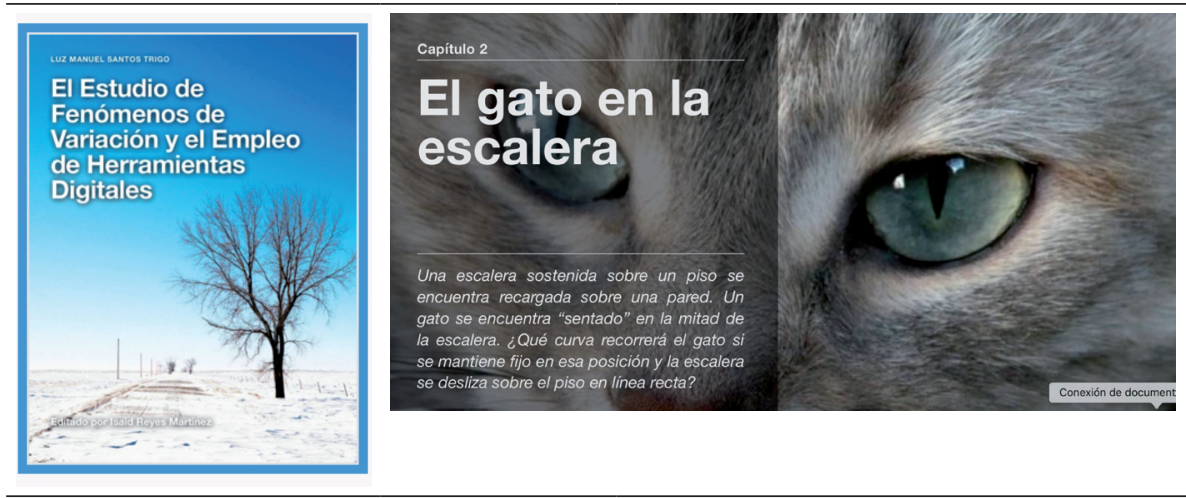

2 Diseñado en el entorno que facilita Apple

3 Santos Trigo \& Reyes-Martínez, 
La Resolución de Problemas Matemáticos y el Uso de Tecnología Digital en el Diseño de Libros Interactivos

Manuel Santos-Trigo y Matías Camacho-Machín

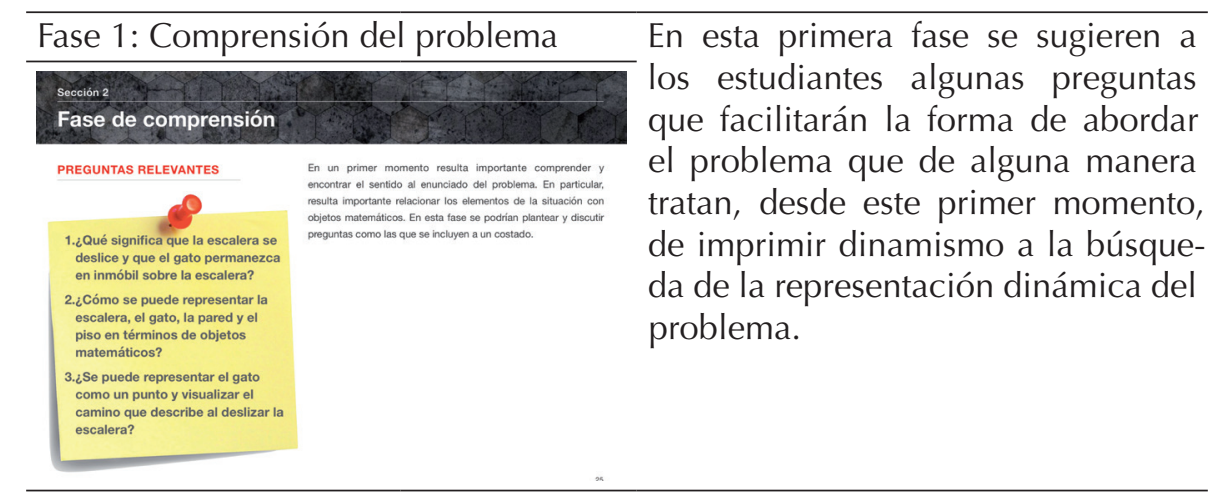

Fase 2: Construcción de un modelo dinámico

\section{La construcción de un modelo matemático}
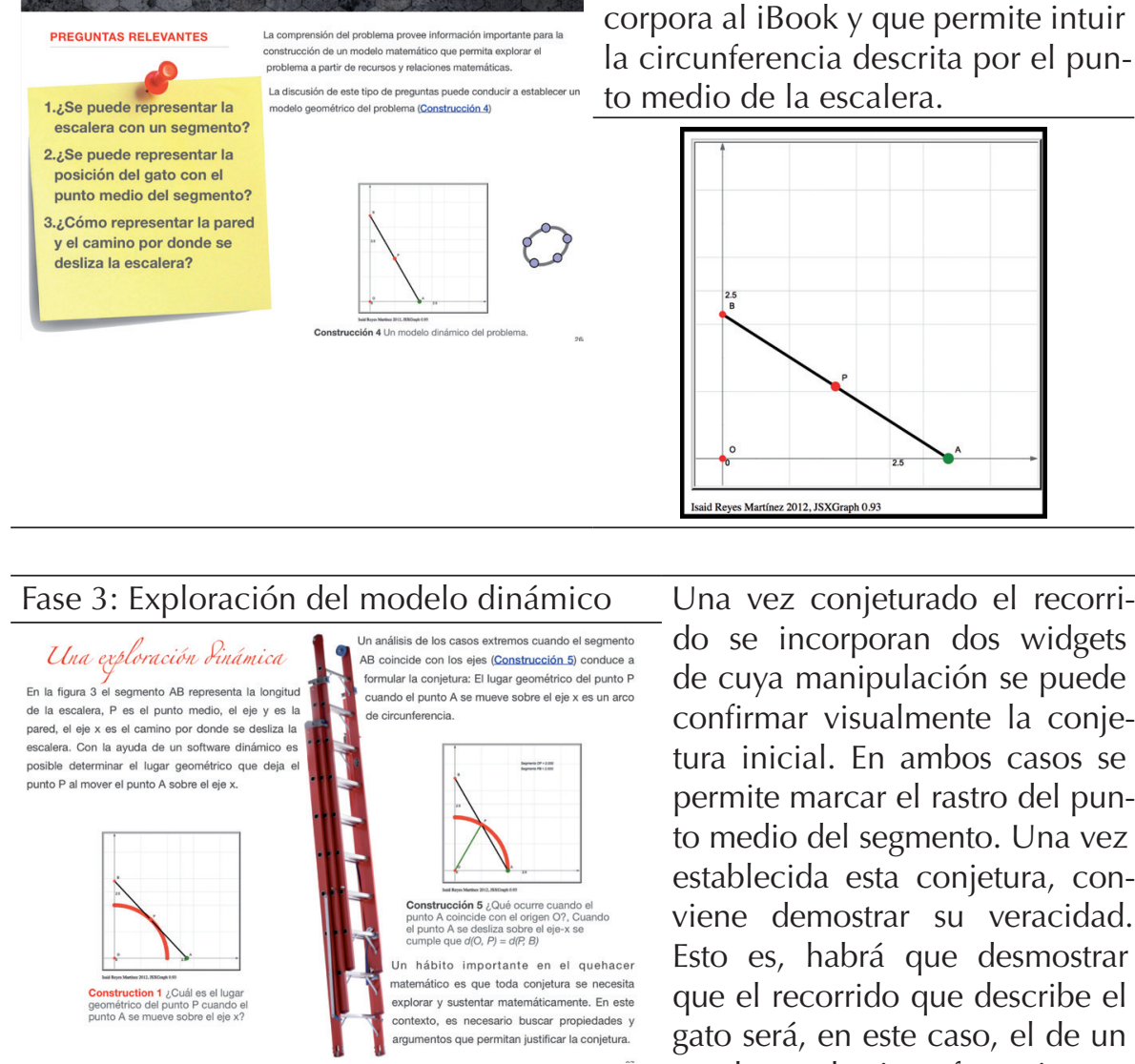

Una vez conjeturado el recorrido se incorporan dos widgets de cuya manipulación se puede confirmar visualmente la conjetura inicial. En ambos casos se permite marcar el rastro del punto medio del segmento. Una vez establecida esta conjetura, conviene demostrar su veracidad. Esto es, habrá que desmostrar que el recorrido que describe el gato será, en este caso, el de un cuadrante de circunferencia 
La Resolución de Problemas Matemáticos y el Uso de Tecnología Digital en el Diseño de Libros Interactivos

Manuel Santos-Trigo y Matías Camacho-Machín

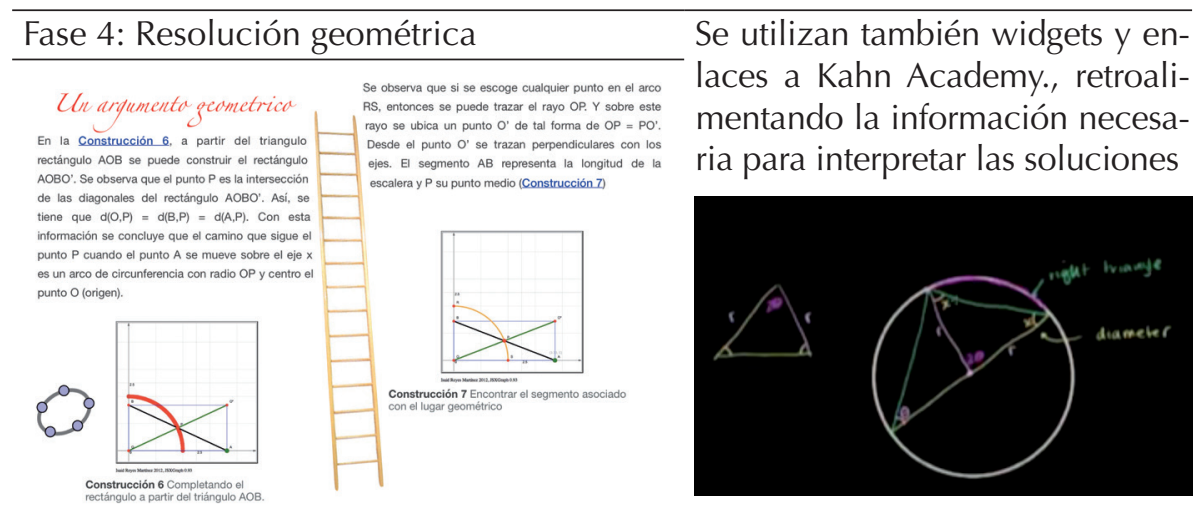

Fase 4: Resolución algebraica

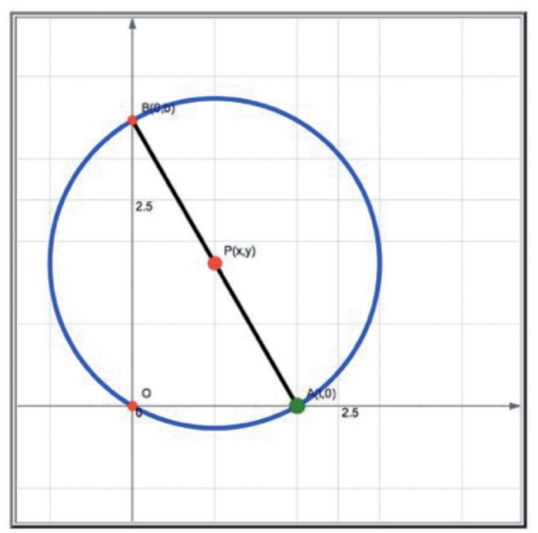

Isaid Reyes Martínez 2012, JSXGraph 0.93
Después de definir las ecuaciones paramétricas del punto medio se determina la circunferencia a la que da lugar

1. $P$ es el punto medio de $A B$, entonces $x=2 / t, y=b / 2$

2. En el triangulo rectángulo $A O B$, se tiene que $t^{\wedge} 2+b^{\wedge} 2$ $=\mathrm{k}^{\wedge} 2 \mathrm{y}$ al sustituir los valores de $\mathrm{x}$ e $\mathrm{y}$, se tiene que $(2 x)^{\wedge} 2+(2 y)^{\wedge} 2=k 2$ lo cuál puede escribirse como $x^{\wedge} 2$ $+y^{\wedge} 2=(k / 2)^{\wedge} 2$. Esta ecuación representa un círculo con radio $\mathrm{k} / 2$ y centro el origen. En términos del problema, el domino y rango de la expresión es el [0, $\mathrm{k} /$ 2].
Fase 5: Extensiones y conexiones

\section{Extensiones y conexiones}

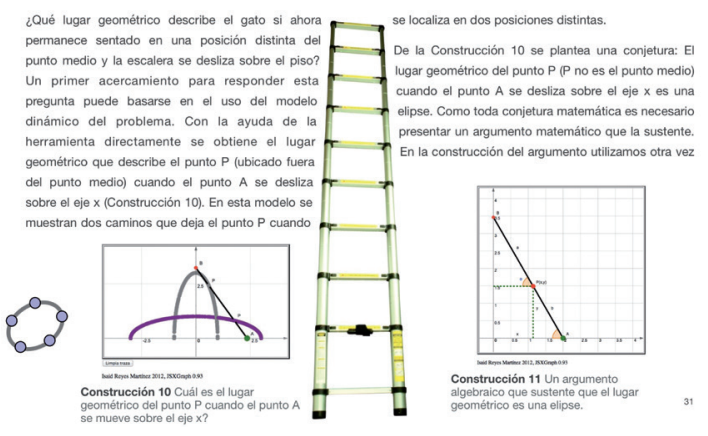

¿Qué lugar geométrico describirá el gato, si permanece sentado en una posición distinta? Se observa, manipulando la widget, que aparentemente se obtendrán elipses. No obstante, para confirmar dicha conjetura, será necesario encontrar los argumentos algebraicos que nos permiten confirmar la conjetura. En la construcción del argumento se utiliza un sistema cartesiano. 
La Resolución de Problemas Matemáticos y el Uso de Tecnología Digital en el Diseño de Libros Interactivos

Manuel Santos-Trigo y Matías Camacho-Machín

De la Construcción 11 se tiene que $\mathrm{P}$ se ubica en el segmento $A B$ y $a+b=d(A, B)$. El ángulo $\widehat{A O B}=\alpha$ representa la inclinación del segmento $A B$ con respecto al eje $x$ $\left(0 \leq \alpha \leq \frac{\pi}{2}\right)$. Con esta información se tiene que, $\sin \alpha=\frac{y}{b}$ y $\cos \alpha=\frac{x}{a}$ lo que lleva a la ecuación: $\frac{x^{2}}{a^{2}}+\frac{y^{2}}{b^{2}}=1$ que representa una elipse.

Se incluyen al final del libro algunos de los conceptos que se consideran relevantes.

\section{Conceptos relevantes}

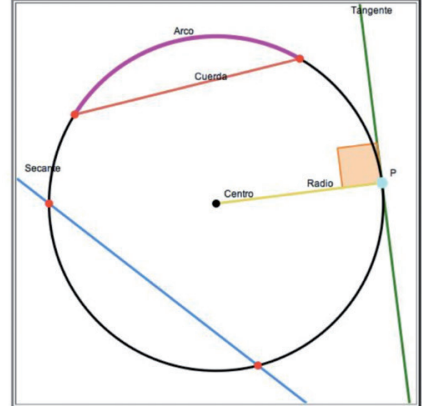

Isaid Reyes Martinez 2012, JSXGraph 0.93

Construcción 13 Elementos de la circunferencia Se puede interactuar con algunos de los puntos, segmentos y rectas notables de la circunferencia.

\section{Ecuación de la circunferencia}

En un sistema de coordenadas cartesianas $x-y$, la circunferencia con centro en el punto $(a, b)$ y radio $r$ consta de todos los puntos $(x, y)$ que satisfacen la ecuación: $(x-h)^{2}+(y-k)^{2}=r^{2}$.

Cuando el centro está en el origen $(0,0)$, la ecuación anterior se simplifica a: $x^{2}+y^{2}=r^{2}$.

La circunferencia con centro en el origen y de radio la unidad, es llamada circunferencia goniométrica, circunferencia unidad o circunferencia unitaria.

De la ecuación general de una circunferencia, $(x-h)^{2}+(y-k)^{2}=r^{2}$, se deduce: $x^{2}+y^{2}+D x+E y+F=0$, resultando:

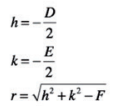

Si conocemos los puntos extremos de un diámetro: $\left(x_{1}, y_{1}\right),\left(x_{2}, y_{2}\right)$, la ecuación de la circunferencia es: $\left(x-x_{1}\right)\left(x-x_{2}\right)+\left(y-y_{1}\right)\left(y-y_{2}\right)=0$.

\section{Algunas consideraciones finales}

En este artículo hemos presentado una serie de elementos de un marco conceptual que pretende guiar a los profesores en el desarrollo y diseño de materiales o libros de texto interactivos, mediante la incorporación 
de actividades de resolución de problemas haciendo uso de tecnologías digitales. En el primer libro interactivo, se ha constatado la importancia de construir modelos dinámicos del problema y siempre buscar diversas maneras de explorarlo y resolverlo. El movimiento y el análisis del comportamiento de algunos elementos del modelo resultaron fundamentales en el camino de conectar la solución del problema con otros objetos matemáticos como la mediatriz y las secciones cónicas básicas: parábola, elipse y la hipérbola. También, durante la exploración de los modelos dinámicos del problema, el estudiante puede consultar otras fuentes o recursos con la intención de revisar o extender su propio dominio de los conceptos. Esta primera fase de análisis del problema resultó importante para el diseño del libro interactivo. Como se muestra en la figura 10 los episodios desarrollados en el análisis de la trayectoria de resolución resultaron ser muy importantes para el proceso de resolución del problema, así como para responder a las preguntas iniciales que motivan la propio resolución. El otro diseño involucra el uso de una tableta iPad, siguiendo la misma idea de buscar y analizar diversas maneras de resolver un problema e integrar otras herramientas que permitan consultar o revisar otros desarrollos en línea durante el proceso de resolución. En este contexto, surgen nuevas preguntas de investigación relacionadas con el diseño, la presentación de las tareas, la evaluación y seguimiento del trabajo de los estudiantes y la selección y uso coordinado de distintas tecnologías en el proceso de resolución de problemas. Además, con el uso de tecnologías digitales los escenarios de enseñanza se amplían y ello demanda que el profesor desarrolle recursos y se apropie de las herramientas que le permitan no sólo caracterizar las formas de razonamiento que construyen los estudiantes, sino también establecer diversas formas de evaluar los acercamientos individuales y grupales que los estudiantes muestren al trabajar y resolver los problemas.

\section{Agradecimientos}

Este trabajo ha sido cofinanciado por los proyectos de investigación de referencias: EDU2017-84276-R del Plan Nacional de Investigación del MINECO y el Proyecto de referencia Conacyt-168543 de México. 
La Resolución de Problemas Matemáticos y el Uso de Tecnología Digital en el Diseño de Libros Interactivos

Manuel Santos-Trigo y Matías Camacho-Machín

\section{Referencias}

Johnson, H. L., Coles, A. \& Clarke, D. (2017). Mathematical tasks and the student: Navigating "tensions of intentions" between designers, teachers, and students. ZDM Mathematics Education, 49: 813-822. DOI 10.1007/s11858-017-0894-0

Leung, A. (2008). Dragging in a dynamic geometry environment through the lens of variation. Int J Comput Math Learning, 13: 135-157. DOI 10.1007/s10758-008-9130-x

Leung, A. (2017). Exploring techno-pedagogic task design in the mathematics classroom. En A. Leung \& A. Baccaglini-Frank (eds.), Digital technologies in designing mathematics education tasks, pp. 3-16. DOI 10.1007/978-3-319-43423-0. Switzerland: Springer.

Howson, G. (2013) The development of mathematics textbooks: historical reflections from a personal perspective. ZDM Mathematics Education, 45:646-658. DOI 10.1007/s11858-013-0511-9.

Santos-Trigo, M. (2014a). Problem solving in mathematics education. En S. Lerman (ed.), Encyclopedia of Mathematics Education (pp. 496-501). NY: Springer.

Santos-Trigo, M. \& Reyes, I. (2016) El Estudio de fenómenos de variación y el empleo de herramientas digitales. https://itunes.apple.com/do/book/interactive-problem-solving-activity-cat-ladder/id688897073? $\mathrm{mt}=11$

Santos-Trigo, M y Camacho-Machín, M. (2013). Framing the use of technology in problem solving approaches. The Mathematics Enthusiast, 10(1-2), 279-302

Santos-Trigo, M. \& Reyes-Martínez, I. (2014). The coordinated use of digital technology in learning environments. In L. Uden et al. (Eds.), Learning Technology for Education in Cloud (pp. 61-71). Communication in Computer and Information Science, 446. NY: Springer.

Schoenfeld, A. H. \& Kilpatrick, J. (2008). Toward a theory of proficiency in teaching mathematics. In T. Wood \& D. Tirosh (eds.), International handbook of mathematics teacher education: Vol. 2. Tools and processes in mathematics teacher education, pp: 321-354. Rotterdam, The Netherlands: Sense Publishers.

Watson, A. \& Ohtani, M. (2015). Themes and issues in mathematics education concerning task design: Editorial introduction. En, A. Watson \& M. Ohtani (eds.), Task design in mathematics education, pp. 3-15: DOI 10.1007/978-3-319-09629-2_1. London: Springer. 